\title{
Estereotipos de los docentes de segundo grado de primaria sobre los perfiles de los estudiantes con alto y bajo rendimiento
}

\author{
Andrés Burga \\ Universidad de Lima \\ Vanessa Sánchez \\ Ministerio de Educación del Perú
}

Recibido: 27 de mayo de 2014 / Aprobado: 24 de junio de 2014

Este estudio buscó identificar los estereotipos que presentan los docentes de segundo grado de primaria que participaron de la Evaluación Censal de Estudiantes 2010 sobre los perfiles de los estudiantes con alto y bajo rendimiento. Para describir a los estudiantes con alto rendimiento, el 40,7\% de docentes utiliza rasgos positivos de manera casi exclusiva. Asimismo, casi el $21 \%$ de docentes emplea rasgos positivos y categorías de urbanidad-costa. En cambio, para describir a los estudiantes con bajo rendimiento, el 30,4\% de docentes usa rasgos negativos. Sin embargo, hay un grupo importante, de $26 \%$, que no suele emplear ninguna de las categorías. La distribución de los docentes según el área geográfica donde se ubica la institución educativa presenta diferencias estadísticamente significativas.

perfiles de rendimiento académico / segundo grado de primaria / área urbano-rural

\section{Second grade teachers' stereotypes on high and low academic perfor- mance student profiles}

The objective of this research was to identify stereotypes that second grade teachers attribute to the profiles of students with high and low academic performance who participated in the 2010 Student Census Evaluation. Results show that 40,7\% of teachers use almost exclusively positive traits to describe students with high performance. Moreover, $21 \%$ of teachers use positive traits and categories related to urban-coastal areas. To describe low performance students, $30,4 \%$ of teachers use negative traits. A significant number of teachers, $26 \%$, however, use neither of the mentioned categories. The geographical areas where schools are located are statistically significant.

academic performance profiles / second grade / urban-rural areas 
El objetivo de este estudio es identificar los estereotipos que presentan los docentes de segundo grado de primaria sobre los perfiles de sus estudiantes con alto y bajo rendimiento académico, diferenciando, además, la distribución de los docentes en cada perfil, según la zona geográfica en la cual se encuentra la institución educativa (IE), ya sea urbana o rural. Para realizar esta investigación, se utilizó información de la Evaluación Censal de Estudiantes (ECE) llevada a cabo en 2010. La ECE es una evaluación a nivel de sistema que realiza anualmente el Ministerio de Educación a través de la Unidad de Medición de la Calidad Educativa (UMC), con el objetivo de obtener información sobre el rendimiento de los estudiantes de segundo y cuarto grado de primaria que trabajan en el marco del Programa de Educación Intercultural Bilingüe (para mayor información sobre las evaluaciones censales, acceda al siguiente enlace del sitio web de la UMC: http://www2.minedu.gob.pe/um c/ece/Marco_de_Trabajo_ECE.pdf).

\section{ESTEREOTIPOS}

Mazzara (1992) indica que en 1922 Walter Lippmann introdujo el término estereotipo en las ciencias sociales, en una publicación sobre procesos de formación de opinión pública. Al analizar sus aportes, se evidencia que Lippmann fue capaz de anticipar puntos esenciales al señalar que los estereotipos forman parte de la cultura de grupo y, como tales, son adquiridos por los individuos y utilizados para una comprensión eficaz de la realidad. Asimismo, para Mazzara, algunas características importantes acerca del estereotipo son su nivel de generalización y el hecho de que son socialmente compartidos, por lo que resultan comunes en el ámbito de ciertas culturas.

Los estereotipos son procesos cognitivos que permiten que las personas se adapten al medio. Anteriormente, tenían una connotación negativa, pero hoy se considera que pueden ser positivos o negativos (Ríos, 2006). No obstante, se debe considerar que los estereotipos que sostenemos de grupos diferentes, a menudo, no son evaluativamente neutros, sino que están sesgados en determinadas direcciones.

De acuerdo con Morales et al. (2007), existe consenso en la literatura con respecto a que los estereotipos son esquemas cognitivos, es decir, son estructuras de datos para representar conceptos genéricos almacenados en la memoria. Los esquemas se utilizan para reflejar conceptos generalizados subyacentes a los objetos, situaciones, sucesos, secuencias de sucesos, acciones y secuencias (Rumelhart \& Ortony, 1982). El autor resume los diferentes aportes describiéndolos como creencias compartidas sobre un grupo, que sirven como ahorro de energía para explicar la realidad social. 
Para Morales (1994), los estereotipos son constructos cognitivos referidos a atributos personales de un grupo social, y aunque con mayor frecuencia estos tienen que ver con rasgos de personalidad, no solo se refieren a ellos. Además, de acuerdo con el autor, se trata de generalizaciones que ignoran en parte la variabilidad de los miembros de un grupo y que tienen un carácter resistente al cambio; así, las creencias estereotípicas se caracterizan por la simplificación o exageración (dado que se basan en casos extremos y salientes), por el carácter consensual (al ser creencias compartidas) y por la rigidez (son difíciles de cambiar).

El estereotipo aparece como parte de los procesos cognitivos de categorización, puesto que permite evocar datos concretos o estereotipados que guían nuestras expectativas. De acuerdo con Medin y Rips (2005), la categorización es un proceso en el cual las representaciones mentales (conceptos) determinan si una entidad es miembro de una categoría. Este proceso hace posible una amplia variedad de funciones subordinadas, pues clasificar algo como miembro de una categoría permite al individuo enfrentar el conocimiento que tiene - acerca de esta categoría- con respecto a otros casos. Una vez que las personas categorizan nuevos casos, pueden usar este conocimiento relevante para entender y predecir. Además, no solamente categorizan a fin de entender nuevos casos, sino que también usan es- tos nuevos casos para modificar y actualizar sus conceptos. En otras palabras, la categorización apoya el aprendizaje y, principalmente, apoya el razonamiento, los cuales se despliegan en las relaciones con los demás. El estereotipo es parte de los procesos cognitivos de categorización en grupos, y se pone de manifiesto cuando el individuo interactúa en relaciones intergrupales.

\section{Expectativas}

Ahora bien, nuestras creencias y juicios sociales tienen efectos e influyen en la forma en que nos sentimos y actuamos, $\mathrm{y}$, por ello, pueden generar su propia realidad. Cuando las ideas nos conducen a actuar en formas que aparentemente las confirman, se convierten en lo que el sociólogo Robert Merton, en 1948, llamó "profecía autocumplida", es decir, son falsas ideas que conducen a su propia realización (Myers, 2005). En este sentido, el fenómeno de la "profecía autocumplida" o "efecto Pigmaleón" en el aula ha sido planteado con el fin de comprender mejor cómo las expectativas de los maestros pueden influir en los estudiantes (Rosenthal \& Jacobson, 1968, 1980). Jussim (1986) desarrolla tres estadios secuenciales que ayudan a explicar cómo funciona la "profecía autocumplida":

a. Los maestros desarrollan expectativas.

b. Los maestros tratan a sus estudiantes de manera diferente, dependiendo de sus expectativas. 
c. Los estudiantes reaccionan a este trato de maneras que confirman las expectativas.

Cabe precisar que, según Jussim, cada una de estas etapas trae consigo una gama de complejos procesos psicológicos y sociales. Además, según este modelo, "el feedback, expectativas, conductas y actitudes del maestro no afectan por igual a todos los alumnos, ya que hay que tener en cuenta también la interpretación del alumno" (García \& Musitu, 1993, p. 60).

Con relación a las expectativas del maestro respecto de sus alumnos, las revisiones que realizaron Brophy y Good (1974), así como Kash et al. (1976), concluyen que los profesores se forman expectativas de sus alumnos y que no tratan a todos de igual manera. Además, estos autores señalan que las expectativas del maestro influyen en cómo este se comporta con el alumno, y en el hecho de que alumnos en la misma clase tengan experiencias diferentes. Otros autores (Frericks, 1974; Burns, 1982) refieren que las expectativas son muy resistentes al cambio, y sus efectos dependen, entre otros aspectos, del nivel de autoestima inicial de los alumnos, del grado en que el profesor es una persona significativa para ellos y de la edad de los mismos.

De acuerdo con Brown (1995), las expectativas estereotípicas podrían llevar al observador a conclusiones erróneas al sesgar la búsqueda y la receptividad hacia la información nueva. Sin embar- go, también podrían ser muy funcionales al permitir percibir algunas cosas muy rápidamente o al liberar recursos cognitivos para concentrarlos en otros asuntos más urgentes. Macrae, Bodenhausen, Milne y Jetten (1994) mostraron a través de diversos experimentos que, a pesar de que exista una represión inicial del estereotipo (por ejemplo, bajo el pedido expreso de evitarlos), este indefectiblemente rebota a la conciencia de las personas. De esta manera, las ideas estereotípicas serían accesibles e influirían fuertemente en las ideas que tienen las personas sobre los grupos. Los mismos autores señalan que los estereotipos repercuten en nuestras expectativas de futuro y, además, de acuerdo con Hamilton y Rose (1980), también pueden sesgar nuestros recuerdos del pasado.

Diversos estudios han demostrado cómo los estereotipos afectan a los juicios, aun cuando la información de las características individuales de la persona juzgada se encuentra disponible (Grant \& Holmes, 1981; Locksley et. al., 1982; Glick et. al., 1988; citados en Brown, 1995). Darley y Gross (1983) concluyeron que no usamos los estereotipos de forma indiscriminada o inconsciente, sino más bien que los estereotipos sirven como hipótesis provisionales para las cuales buscamos información posterior; incluso, como señala Brown (1995), es posible que la gente busque información para confirmar sus hipótesis. 


\section{Expectativas y rendimiento académico}

Existe información empírica consistente con respecto a los efectos de las expectativas de los maestros sobre el rendimiento del estudiante, a partir de estudios experimentales y naturalistas (Rosenthal \& Rubin, 1978; Smith, 1980; Brophy, 1983; Brattesani et al., 1984; Raudenbush, 1984; Dusek, 1985; Babad, 1993; Jussim \& Eccles, 1995; citados en Alvidrez \& Weinstein, 1999). Sin embargo, algunos investigadores sugieren que los grandes efectos se relacionan más con los estudiantes que con los maestros, y que los efectos de las expectativas que resultan del sesgo en la percepción son relativamente pequeños (representan cerca del 5 $\%$ y el $10 \%$ de la varianza con relación al logro del estudiante) (Brophy, 1983; Jussim, 1989, 1991; Jussim \& Eccles, 1992; citados en Alvidrez y Weinstein, 1999). Además, la literatura también señala que las altas correlaciones entre las expectativas de los maestros y el logro de los estudiantes encontradas en muchos estudios (por ejemplo, en los de Brophy \& Good, 1974; Humphreys \& Stubbs, 1977; Crano \& Mellon, 1978; citados en Jussim, 1986) incorporan tanto los efectos de la "profecía autocumplida" como la precisión de las expectativas con respecto a los estudiantes.

Darley y Gross (1983) hallaron que los estereotipos de clase social pueden influir en los juicios que se forman los maestros acerca del rendimiento académico de sus estudiantes. Dusek y
Joseph (1983) encuentran que las expectativas de los maestros por niños de minorías étnicas o pertenecientes a grupos socioeconómicos bajos son, generalmente, más bajas. Estudios en los cuales las diferencias de logro entre los estudiantes han sido controladas muestran que estas expectativas podrían reflejar sesgos. Así, en un metaanálisis de estudios experimentales sobre las expectativas de los maestros, Baron, Tom y Cooper (1985) encontraron que el juicio de los maestros con respecto a los estudiantes blancos de clase media fue más favorable que para aquellos afroamericanos pertenecientes a niveles socioeconómicos bajos, ello a pesar de que ambos grupos tenían un logro similar. Sin embargo, otros estudios naturalistas (Haller, 1985) y revisiones (Jussim, Madon \& Chatman, 1994) no muestran evidencia de la existencia de sesgos de origen étnico en la percepción de la habilidad estudiantil.

Según Oakes (1985), una de las razones por las cuales los maestros de estudiantes más pobres o pertenecientes a minorías los ubican más bajo que a otros estudiantes es debido a la existencia de un "currículum oculto", en el cual las expectativas y los juicios de los maestros se basan en la presencia de características conductuales sutiles que el niño trae de la vida en el hogar y muestra en el aula de clase. Fischer y Thomas (1965) señalan la existencia de particularidades en las subculturas —que incluyen aspectos del lenguaje 
y la conducta-, las cuales tienen una influencia en el estilo de aprendizaje de los niños y, por ende, en la manera como los niños aprenden.

Según otros estudios, la valoración del maestro con respecto al logro del estudiante también se relaciona con el atractivo físico y con ciertas conductas en el aula de clases, tales como la atención, el esmero y la independencia que se demuestra en el aula (Dusek \& Joseph, 1983; Pedulla, Airasian \& Madaus, 1980).

Finalmente, en el Perú, una investigación realizada como parte de una tesis universitaria estudió las actitudes y estereotipos raciales que presentaban los profesores de primaria y secundaria del distrito de Comas, en Lima (Ríos, 2006). Algunos de los resultados encontrados señalan que los maestros de primaria y secundaria valoran más a las razas blanca y amarilla (percibidas como inteligentes), mientras que la negra e india son menos valoradas (descritas como sucias); también se evidencia una tendencia ascendente en la valoración del "cholo" respecto a estudios anteriores. Además, se observa una contradicción en los centros educativos entre el discurso de igualdad impartido y las actitudes negativas de sus docentes hacia algunos grupos raciales (cholo, indio y negro). Los profesores nacidos en la costa fuera de Lima desvalorizan al indio, negro y amarillo; en cambio, los nuevos migrantes desvalorizan a los blancos.

\section{Método}

\section{Participantes}

Esta investigación se llevó a cabo en el marco de la Evaluación Censal de Estudiantes 2010 (ECE 2010), coordinada por la Unidad de Medición de la Calidad Educativa (UMC) del Ministerio de Educación del Perú (MED). Como parte de este operativo, se toma una muestra control (MC) de instituciones educativas (IE) en las cuales se aplican procedimientos más rigurosos, para asegurar que los resultados a nivel nacional y regional tengan la precisión deseada, controlando errores no muestrales, como la baja cobertura o la aplicación incorrecta de los instrumentos de recolección de datos. En este estudio se analizan los datos obtenidos de dicha muestra control.

La muestra control para la ECE 2010 está conformada por 5085 instituciones educativas. Para asegurar una dispersión adecuada en la muestra, se realizó una estratificación por gestión (estatal, no estatal), área (urbana, rural) y característica (unidocente/multigrado, polidocente completo) dentro de cada una de las 26 regiones (se considera Lima Provincias y Lima Metropolitana como regiones).

El método de muestreo para la selección de la IE fue aleatorio sistemático, tomando como marco muestral el listado de IE que poseen cinco o más estudiantes en segundo grado de primaria, según lo reportado por la Unidad de 
Tabla 1

Cantidad de participantes diferenciados por departamento, gestión de la IE y área geográfica donde esta se ubica $(N=5874)$

\begin{tabular}{|c|c|c|c|c|}
\hline \multirow{2}{*}{ Región } & \multicolumn{2}{|c|}{ Estatal } & \multicolumn{2}{|c|}{ No estatal } \\
\hline & Urbana & Rural & Urbana & Rural \\
\hline Amazonas & 75 & 99 & 1 & 0 \\
\hline Áncash & 117 & 142 & 32 & 1 \\
\hline Apurímac & 51 & 73 & 5 & 0 \\
\hline Arequipa & 104 & 20 & 89 & 0 \\
\hline Ayacucho & 37 & 53 & 8 & 0 \\
\hline Cajamarca & 89 & 263 & 16 & 12 \\
\hline Callao & 52 & 0 & 65 & 0 \\
\hline Cusco & 89 & 120 & 26 & 3 \\
\hline Huancavelica & 34 & 81 & 2 & 0 \\
\hline Huánuco & 72 & 203 & 15 & 1 \\
\hline Ica & 66 & 18 & 41 & 1 \\
\hline Junín & 105 & 139 & 46 & 2 \\
\hline La Libertad & 114 & 187 & 79 & 0 \\
\hline Lambayeque & 51 & 51 & 53 & 2 \\
\hline Lima Provincias & 97 & 43 & 36 & 1 \\
\hline Lima Metropolitana & 224 & 0 & 347 & 1 \\
\hline Loreto & 101 & 254 & 14 & 3 \\
\hline Madre de Dios & 62 & 39 & 14 & 0 \\
\hline Moquegua & 106 & 9 & 32 & 0 \\
\hline Pasco & 56 & 37 & 4 & 0 \\
\hline Piura & 135 & 179 & 60 & 1 \\
\hline Puno & 63 & 73 & 22 & 3 \\
\hline San Martín & 123 & 178 & 7 & 2 \\
\hline Tacna & 141 & 21 & 70 & 2 \\
\hline Tumbes & 132 & 31 & 28 & 0 \\
\hline Ucayali & 64 & 51 & 3 & 0 \\
\hline TOTAL & 2360 & 2364 & 1115 & 35 \\
\hline
\end{tabular}

Fuente: base de datos de la ECE 2010. 
Estadística Educativa del MED. Dicho marco muestral contiene 24762 instituciones educativas que congregan a 604 896 alumnos.

Si bien la ECE 2010 tiene como unidad de análisis al estudiante, en este informe se ha trabajado con el docente como unidad de análisis. Del conjunto de 5085 IE se logró recolectar información sobre 5874 docentes que se distribuyeron como se indica en la tabla 1.

El $73,1 \%$ de participantes son de sexo femenino y el $26,9 \%$ son de sexo masculino. La edad promedio del grupo femenino es 39,8 años $(D E=9,2)$ y la del masculino 42,1 (DE = 9,5).

\section{Instrumento}

El instrumento utilizado en este reporte de investigación fue aplicado como parte de los cuestionarios de factores asociados de la ECE 2010. Las evidencias de validez del instrumento referidas a su contenido provienen del uso del criterio de jueces. Un grupo de seis personas con experiencia en investigación educativa y/o docencia en aula revisaron las 15 características descritas en este instrumento y, por unanimidad, concluyeron que estas pueden ser utilizadas para describir características típicas de los estudiantes con alto o bajo rendimiento.

El instrumento fue presentado a los participantes a manera de una tabla de doble entrada. Las filas pre- sentaban el tipo de estudiante (con alto o bajo rendimiento) y las columnas, las características que podrían ser asociadas a dichos estudiantes. Estas fueron ordenadas de manera aleatoria en el cuestionario, donde aparecían en las columnas en el siguiente orden: es inteligente, es ordenado, es descuidado en su aspecto personal, es disciplinado, tiene padres separados, viene de la costa, es responsable, empezó la escuela con extraedad, viene de un medio rural, es inquieto, viene de un medio urbano, viene de la selva, molesta a sus compañeros, viene de la sierra, tiene una lengua distinta al castellano.

La instrucción presentada a los participantes fue la siguiente: "Según su opinión, señale qué características tienen los estudiantes con alto y bajo rendimiento. Puede marcar varias respuestas en cada fila". Dichas marcas fueron realizadas con lápiz, para rellenar la burbuja de la ficha óptica que correspondía a cada celda en la que se interceptaba una fila con una columna.

\section{Estrategias de análisis de los resultados}

Las estrategias de análisis implicaron trabajar, de manera independiente, el perfil del estudiante con alto y bajo rendimiento. En ambos casos se aplicó el análisis de componentes principales para datos categóricos (Linting \& Van der Kooij, 2012) con el módulo CATPCA de 
IBM SPSS Statistics v. 20. Este análisis se realizó sobre las cuantificaciones de las categorías dicotómicas de respuesta, usando luego la rotación varimax y manteniendo los ítems con cargas superiores a 0,3 en un componente.

Usando los puntajes de los docentes en cada componente, se aplicó la técnica de conglomerados en dos pasos (TwoStep Cluster) para armar perfiles de respuesta, tanto en el caso del estudiante con alto rendimiento como en el del que presenta bajo rendimiento. Analizando las diferencias en las puntuaciones promedio en cada uno de los cuatro componentes en cada conglomerado estimado, se describieron los grupos de docentes en función del perfil que utilizan para caracterizar a los estudiantes con alto y bajo rendimiento.

Finalmente, se aplicó la prueba $F$ corregida, variante del estadístico chicuadrado de independencia proporcionada por el módulo de muestras complejas de IBM SPSS Statistics v. 20., para analizar la presencia de diferencias estadísticamente significativas en las proporciones de docentes en cada tipo de perfil encontrado, según la ubicación geográfica de las IE.

\section{Resultados}

En la tabla 2 presentamos el porcentaje de docentes que asoció cada una de las características presentadas con los estudiantes con alto y bajo rendimiento.
Los datos de los docentes fueron sometidos al CATPCA y se extrajeron cuatro componentes que explican el 49 $\%$ de la varianza en el perfil de alto rendimiento y el $48 \%$ en el de bajo rendimiento (ver tabla 3 y tabla 4). En ambos casos se requirieron seis iteraciones para la convergencia de los resultados. A continuación, se muestra la matriz de cargas en los componentes extraídos tanto en el caso del perfil del estudiante con alto rendimiento como en el de aquel con bajo rendimiento.

$\mathrm{Al}$ analizar el contenido de los ítems que presentan las mayores cargas en cada componente, se decidió denominarlos de la siguiente manera:

- Rasgos positivos: inteligente, ordenado, disciplinado, responsable.

- Rasgos negativos: descuidado en aspecto personal, inquieto, molesta a sus compañeros, padres separados.

- Urbanidad-costa: urbano, de la costa.

- Ruralidad-no costa: viene de medio rural, viene de la selva, viene de la sierra, lengua distinta al castellano.

Para finalizar el análisis del instrumento, se calculó el coeficiente alfa de Cronbach de cada uno de los componentes, el cual se presenta en la tabla 5.

Aplicando el análisis de conglomerados, se diferenciaron los distintos perfiles que utilizarían los docentes para describir a los estudiantes con alto y bajo rendimiento. Los porcentajes de docen- 
Tabla 2

Porcentaje de elección de cada una de las categorías para describir al estudiante con alto y bajo rendimiento

\begin{tabular}{lrccc}
\hline \multirow{2}{*}{ Categoría } & \multicolumn{2}{c}{ Alto rendimiento } & \multicolumn{2}{c}{ Bajo rendimiento } \\
\cline { 2 - 5 } & $\%$ & e. e. & $\%$ & e. e. \\
\hline Inteligente & 82,8 & 0,5 & 10,4 & 0,4 \\
Ordenado & 81,0 & 0,5 & 11,2 & 0,4 \\
Aspecto descuidado & 9,0 & 0,4 & 65,0 & 0,7 \\
Disciplinado & 70,9 & 0,6 & 12,7 & 0,5 \\
Padres separados & 9,2 & 0,4 & 65,3 & 0,7 \\
Costa & 17,5 & 0,6 & 10,3 & 0,4 \\
Responsable & 86,5 & 0,5 & 5,9 & 0,3 \\
Extraedad & 7,4 & 0,4 & 23,8 & 0,6 \\
Rural & 21,6 & 0,6 & 32,4 & 0,7 \\
Inquieto & 43,6 & 0,7 & 51,5 & 0,7 \\
Urbano & 25,5 & 0,6 & 11,3 & 0,4 \\
Selva & 7,7 & 0,4 & 11,7 & 0,5 \\
Molesta compañeros & 13,0 & 0,5 & 61,6 & 0,7 \\
Sierra & 13,9 & 0,5 & 15,6 & 0,5 \\
Lengua originaria & 9,4 & 0,4 & 16,8 & 0,5 \\
\hline
\end{tabular}

Fuente: Base de datos de la ECE 2010.

Nota: e. e. corresponde al error estándar.

tes en cada perfil se encuentran ponderados, considerando la distribución nacional por regiones, el tipo de gestión de la IE (estatal o no estatal) y la zona donde se ubica la IE (urbana o rural). Esta ponderación se expande a una población que estima el número total de docentes como equivalente al número de secciones que se esperaba evaluar en la ECE 2010 (es decir: 32 554).

En el caso de las descripciones de los estudiantes con alto rendimiento, se propone una tipología de los docentes que los ubica en uno de los seis grupos que se describen a continuación:

- Grupo 1: utiliza todos los descriptores.

- Grupo 2: utiliza los rasgos negativos y en parte la urbanidad-costa.

- Grupo 3: casi no utiliza ninguna categoría, pero tiende al uso de ruralidad-no costa.

- Grupo 4: utiliza rasgos positivos y urbanidad-costa.

- Grupo 5: utiliza, sobre todo, rasgos positivos, ruralidad-no costa y 
Tabla 3

Carga de cada ítem en los cuatro componentes extraídos luego de la rotación varimax, características del estudiante con ALTO rendimiento

\begin{tabular}{lcccc}
\hline \multirow{2}{*}{ Categoría } & \multicolumn{3}{c}{ Componente } \\
\cline { 2 - 4 } & $\mathbf{1}$ & $\mathbf{2}$ & $\mathbf{3}$ & $\mathbf{4}$ \\
\hline Lengua originaria & 0,716 & & & \\
Rural & 0,670 & & & 0,342 \\
Sierra & 0,669 & & 0,342 \\
Selva & 0,580 & & & \\
Extraedad & 0,420 & & & \\
Responsable & & 0,758 & \\
Ordenado & & 0,735 & \\
Disciplinado & & 0,614 & \\
Inteligente & & 0,606 & 0,725 & \\
Inquieto & & & 0,706 & \\
Molesta compañeros & & 0,514 & \\
Aspecto descuidado & & 0,452 & \\
Padres separados & & & & \\
Urbano & & & & \\
Costa & & & & \\
\hline
\end{tabular}

Fuente: base de datos de la ECE 2010.

emplea de manera moderada rasgos negativos.

- Grupo 6: utiliza, casi exclusivamente, rasgos positivos.

En la tabla 6, se observa una diferencia estadísticamente significativa en la distribución de los docentes en los grupos según la zona donde se ubica la IE en la cual laboran, $F(4.98,22201.54)=143,28$, $p<.001$.

En el caso de las descripciones de los estudiantes con bajo rendimiento, se propone una tipología de los docentes que los ubica en uno de los cinco grupos que se describen a continuación:

- Grupo 1: no suele emplear ninguna de las categorías.

- Grupo 2: utiliza los rasgos negativos.

- Grupo 3: utiliza rasgos positivos.

- Grupo 4: usa, sobre todo, la categoría de urbanidad-costa y de manera moderada el resto de categorías. 
Tabla 4

Carga de cada ítem en los cuatro componentes extraídos luego de la rotación varimax, características del estudiante con BAJO rendimiento

\begin{tabular}{lcccc}
\hline \multirow{2}{*}{ Categoría } & \multicolumn{4}{c}{ Componente } \\
\cline { 2 - 5 } & $\mathbf{1}$ & $\mathbf{2}$ & $\mathbf{3}$ & $\mathbf{4}$ \\
\hline Lengua originaria & 0,687 & & & \\
Rural & 0,674 & & 0,433 & \\
Sierra & 0,643 & & & \\
Selva & 0,602 & & & \\
Extraedad & 0,544 & & & \\
Responsable & & 0,731 & \\
Ordenado & & 0,676 & \\
Disciplinado & & 0,614 & 0,777 \\
Inteligente & & 0,594 & 0,782 \\
Urbano & & & 0,776 \\
Costa & & & & \\
Molesta compañeros & & & & \\
Inquieto & & & & \\
Aspecto descuidado & & & & \\
Padres separados & & & & \\
\hline
\end{tabular}

Fuente: base de datos de la ECE 2010.

- Grupo 5: utiliza, sobre todo, rasgos negativos y ruralidad-no costa.

La distribución de docentes de segundo grado, que pertenecen a cada uno de los grupos según las características que asocian a los estudiantes de bajo rendimiento, se presenta a continuación.

En la tabla 7, se observa una diferencia estadísticamente significativa en la distribución de los docentes en los gru- pos de bajo rendimiento, según la zona en la que se ubica la IE en la cual laboran, $F(3.99,17801.47)=45,08, p<.001$.

\section{DisCusión}

Es importante resaltar que la estructura de componentes principales utilizada para describir tanto al estudiante con alto como bajo rendimiento es similar, lo cual contribuye a validar la estabilidad de los resultados estructurales. En 
Tabla 5

Coeficiente alfa de Cronbach calculado en cada componente, perfiles de ALTO y BAJO rendimiento

\begin{tabular}{ccc}
\hline Componente & Alto rendimiento & Bajo rendimiento \\
\hline Rasgos positivos & 0,64 & 0,59 \\
Rasgos negativos & 0,54 & 0,45 \\
Urbanidad-costa & 0,57 & 0,60 \\
Ruralidad-no costa & 0,66 & 0,69 \\
\hline
\end{tabular}

Fuente: base de datos de la ECE 2010.

Tabla 6

Porcentaje de docentes en cada uno de los perfiles elaborados para describir al estudiante con ALTO rendimiento y diferenciados según zona donde se ubica la IE

\begin{tabular}{ccccccc}
\hline \multirow{2}{*}{ Grupo } & \multicolumn{2}{c}{ Total } & \multicolumn{2}{c}{ Urbana } & \multicolumn{2}{c}{ Rural } \\
\cline { 2 - 7 } & $\%$ & e. e. & $\%$ & e. e. & $\%$ & e. e. \\
\hline 1 & 4,8 & 0,29 & 4,7 & 0,40 & 4,9 & 0,42 \\
2 & 10,9 & 0,43 & 12,1 & 0,60 & 9,0 & 0,57 \\
3 & 10,7 & 0,42 & 7,1 & 0,49 & 16,4 & 0,73 \\
4 & 20,9 & 0,56 & 28,5 & 0,81 & 9,0 & 0,56 \\
5 & 12,0 & 0,44 & 5,3 & 0,38 & 22,6 & 0,86 \\
6 & 40,7 & 0,67 & 42,3 & 0,91 & 38,1 & 0,97 \\
\hline
\end{tabular}

Fuente: base de datos de la ECE 2010.

Nota: e. e. corresponde al error estándar.

la mente de los docentes participantes se perciben como asociadas las mismas categorías que sirven para describir tanto a los estudiantes con alto como bajo rendimiento, indicando que son constructos similares. Si bien el coeficiente alfa de Cronbach estimado para cada componente es bajo dentro de cada perfil, ello puede deberse a que se trata de pocos ítems, que además tienen calificación binaria.
Los resultados muestran que los docentes, en su mayoría, forman estereotipos acerca de cómo son los estudiantes con alto y bajo rendimiento (Morales et al., 2007; Morales, 1994). Al aparecer, cinco perfiles estereotipados sobre los estudiantes de bajo rendimiento y seis sobre los de alto rendimiento nos permiten señalar que hay mayor consenso respecto al primer perfil que sobre el segundo. 
Tabla 7

Porcentaje de docentes en cada uno de los perfiles elaborados para describir al estudiante con BAJO rendimiento, diferenciados según zona donde se ubica la IE

\begin{tabular}{ccccccc}
\hline \multirow{2}{*}{ Grupo } & \multicolumn{2}{c}{ Total } & \multicolumn{2}{c}{ Urbana } & \multicolumn{2}{c}{ Rural } \\
\cline { 2 - 7 } & $\%$ & e. e. & $\%$ & e. e. & $\%$ & e. e. \\
\hline 1 & 26,3 & 0,60 & 22,5 & 0,8 & 32,3 & 0,9 \\
2 & 30,4 & 0,65 & 34,2 & 0,9 & 24,4 & 0,9 \\
3 & 14,3 & 0,46 & 12,6 & 0,6 & 16,8 & 0,7 \\
4 & 12,9 & 0,47 & 15,9 & 0,7 & 8,2 & 0,5 \\
5 & 16,2 & 0,51 & 14,9 & 0,7 & 18,3 & 0,8 \\
\hline
\end{tabular}

Nota: e. e. corresponde al error estándar.

Otro resultado importante es que muy pocos docentes (cerca del $5 \%$ ) reportan que un estudiante con alto rendimiento puede tener cualquier característica. Esto contrasta notablemente con el porcentaje de docentes que señala que cualquier característica puede estar asociada con un estudiante de bajo rendimiento (cerca del $26 \%$ ). El resultado anterior sugiere que es más probable aceptar que cualquier estudiante, independientemente de sus características socioculturales y comportamentales, puede tener un rendimiento bajo, que aceptar que cualquier estudiante puede tener un alto rendimiento. Ello parece estarnos hablando de una visión más bien pesimista sobre el logro estudiantil, cuando se trata de asociarlo con características sociodemográficas y/o comportamentales.

Si los docentes actúan según el estereotipo "a cualquiera le va mal, pero no a cualquiera le va bien", se podría generar una "profecía autocumplida" (Oakes, 1985), que reforzaría los bajos logros de ciertos grupos, pues "no a cualquiera le va bien". Rosenthal (1991) reportó que los maestros observan, sonríen y apoyan más a los "estudiantes con un alto potencial". Harris y Rosenthal (1985), y Jussim (1986) encontraron que los maestros también tienden a enseñar más cosas a sus alumnos "con mayor potencial", a establecer metas más altas, a ponerles mayor atención e incluso a darles más tiempo para responder.

Según se observa, en el caso de las descripciones de los estudiantes con alto rendimiento, el 40,7 \% utiliza casi exclusivamente rasgos positivos. Asimismo, casi el $21 \%$ de docentes utiliza rasgos positivos y selecciona lo referido a urbanidad-costa. Los docentes que utilizan casi exclusivamente rasgos positivos, o los usan en combinación con la urbanidad-costa para caracterizar a estos estu- 
diantes, son en mayor proporción docentes de IE ubicadas en zonas urbanas.

En el caso del perfil de los estudiantes con bajo rendimiento, el 30,4\% usa rasgos negativos para describirlos. Sin embargo, hay un grupo importante de 26 $\%$ de docentes que no suele emplear ninguna de las categorías. Los docentes que usan rasgos negativos para describirlos pertenecen en mayor proporción a las IE ubicadas en zonas rurales. Lo contrario ocurre con los docentes que no suelen emplear ninguna de las categorías; ellos pertenecen en mayor proporción a las IE ubicadas en zonas urbanas.

Los resultados muestran que, en general, los docentes emplean características estereotípicas para describir a sus estudiantes, principalmente aquellas asociadas con rasgos explícitamente positivos o negativos, según la definición de este estudio. Es interesante observar que dichas apreciaciones varían según el área geográfica donde se ubican las IE. En cuanto a las características del estudiante con alto rendimiento, se encuentra que las mayores diferencias en la distribución de los docentes en los distintos grupos, según la zona donde se sitúa la IE, están en los grupos 3 (casi no utiliza ninguna categoría, tiende al uso de ruralidad-no costa), 4 (rasgos positivos y urbanidad-costa) y 5 (rasgos positivos, ruralidad-no costa, uso moderado de rasgos negativos). Estas diferencias son importantes, pues en ambos casos nos estarían hablando de una autovaloración positiva del grupo en cuestión. Si bien Dusek y Joseph (1983) encuentran que las expectativas de los maestros por niños de minorías étnicas o pertenecientes a grupos socioeconómicos bajos son, generalmente, más bajas, es interesante notar que la identificación del estudiante de alto rendimiento usando categorías de ruralidad-no costa ocurre en aproximadamente un $38 \%$ de los docentes cuya IE se encuentra en zonas rurales. Esto nos informa sobre una autoevaluación positiva de este grupo humano, aunque en la mayoría de ellos, además del uso de categorías de ruralidad-no costa, se utilicen rasgos positivos para describirlos.

Analizando las diferencias en los perfiles de bajo rendimiento, las principales diferencias entre los docentes según la zona en la que se ubica la IE se encuentran en los grupos 1 (ninguna categoría predomina), 2 (rasgos negativos) y 4 (urbanidad-costa). En cuanto a los grupos 1 y 2 , se observa un mayor porcentaje del grupo 1 en la zona rural y de los grupos 2 y 4 en la zona urbana. Este resultado indicaría que hay mayores prejuicios, sobre todo los asociados con características negativas, entre los docentes de IE ubicadas en zonas urbanas.

Pasando a otro punto, los resultados del análisis de factores asociados al rendimiento de las Evaluaciones Nacionales de 2001 y 2004 arrojan que existe una relación positiva entre las expectativas de los docentes y el rendimiento estudiantil (UMC del MED en 
2004). Estos resultados también aparecen en la ECE 2010. Además, en ambas evaluaciones se observa que hay un mayor rendimiento en comprensión lectora y matemática, al comparar las IE ubicadas en zonas urbanas con las de zonas rurales. Si se toma en cuenta esta información, el hallazgo acerca de los diferentes perfiles y estereotipos de los docentes puede hacernos suponer que existen expectativas estereotípicas relacionadas con el rendimiento, que en parte estarían contribuyendo a las diferencias de logro antes mencionadas.

La investigación acerca de los perfiles y estereotipos que presentan los docentes sobre los estudiantes con alto y bajo rendimiento permite explorar algunos aspectos subjetivos de la relación que se establece entre el docente y sus estudiantes. Efectivamente, el estudio de las percepciones de los docentes acerca de sus estudiantes es un aspecto sobre el cual es interesante investigar, debido a las implicancias de la conducta del docente en el aula, y específicamente en el rendimiento y aprendizaje de sus estudiantes. El proceso de aprendizaje en el aula tiene en el docente a una figura fundamental, pues a partir de la forma como este desarrolla sus clases y la manera como interactúa con sus estudiantes puede promover diferentes estilos de aprendizaje y oportunidades para ellos (Woolfolk, Woolfolk \& Margetts, 2010).
La valoración de características positivas-negativas atribuidas a los estudiantes ha sido propuesta desde una mirada de investigador. Sin duda, la interacción del profesor con sus estudiantes en el aula, $\mathrm{y}$ la observación de la misma, podría poner en evidencia conductas asociadas a los estereotipos que permitirían conocerlos mejor. Se la tendría que explorar en la práctica en el aula, a fin de conocer en qué medida el "efecto Pigmaleón" se encuentra presente en las expectativas estereotípicas que podrían influir en el alto o bajo rendimiento de los estudiantes.

\section{REFERENCIAS}

Alvidrez, J., \& Weinstein, R. S. (1999). Early Teacher Perception and Later Student Academic Achievement. Journal of Educational Psychology, 91 (4), diciembre, 1999, 731-746.

Baron, R., Tom, D., \& Cooper, H. (1985). Social Class, Race and Teacher Expectations. En J. Dusek (Ed.), Teacher Expectancies (pp. 251-270). Hillsdale, NJ: Lawrence Erlbaum Associates.

Brophy, I., \& Good T. (1974). TeacherStudent Relationships: Causes and Consequences. Nueva York: Holt, Rinehart and Winston.

Brown, R. (1995). Prejuicio: su psicología social. Madrid: Alianza Editorial. Burns, R. (1982). Self-Concept Development and Education. Londres: Holt, Rinehart and Winston. 
Darley, J., \& Gross, P. (1983). A Hypothesis-Confirming Bias in Labeling Effects. Journal of Personality and Social Psychology, 44, 20-33.

Dusek, J., \& Joseph, G. (1983). The Bases of Teacher Expectancies: A meta-analysis. Journal of Educational Psychology, 75, 327-346.

Fischer, L., \& Thomas, D. (1965). Social Foundation of Educational Decisions. Belmont, CA: Wadsworth Publishing Co.

Frericks, A. (1974). Labelling of Students by Prospective Teachers. Paper presented at the American Educational Research Association Convention, Chicago.

García, F., \& Musitu, G. (1993). Un programa de intervención basado en la autoestima: análisis de una experiencia. Revista de Psicología Universitas Tarraconensis, vol. XV (1/1993).

Haller, E. (1985). Pupil Race and Elementary School Ability Grouping: Are Teachers Biased against Black Children? American Educational Research Journal, 22, 465-483.

Hamilton, D., \& Rose, T. (1980). Illusory Correlation and the Maintenance of Stereotypic Beliefs. Journal of Personality and Social Psychology, 39, 832-845.

Harris, M., \& Rosenthal, R. (1985). Mediation of Interpersonally Ex- pectancy Effects: 31 meta-analyses. Psychological Bulletin, 97, 363-386.

Jussim, L. (1986). Self-Fulfilling Prophecies: A Theoretical and Integrative Review. Psychological Review, vol. 93 (4), octubre, 1986, 429-445.

Jussim, L., Madom, S., \& Chatman, C. (1994). Teacher Expectations and Student Achievement: Self-Fulfilling Prophecies, Biases and Accuracy. En L. Health, R. S. Tindale, J. Edwards, E. J. Posavac, F. B. Bryant, E. Henderson-King, Y. SuárezBakazar, \& J. Meyers (Eds.), Applications of Heuristics and Biases to Social Issues (pp. 303-334). Nueva York: Plenum.

Kash, M. et al. (1976). Teacher Behavior and Pupil Self-Concept. Research and Development Center for Teacher Education. Austin: University of Texas.

Linting M., \& Van der Kooij, A. (2012). Nonlinear Principal Components Analysis with CATPCA: A Tutorial. Journal of Personality Assessment, 94 (1), 12-25.

Macrae, C., Bodenhausen, G., Milne, A., \& Jetten, J. (1994). Out of Mind but Back in Sight: Stereotypes on the Rebound. Journal of Personality and Social Psychology, 67 (5), 808-817.

Mazzara, B. (1999). Estereotipos y prejuicios. Madrid: Acento Editorial. 
Medin, D., \& Rips, L. (2005). Concepts and Categories: Memory, Meaning and Metaphysics. En K. Holyoak \& R. Morrison (Eds.), The Cambridge Handbook of Thinking and Reasoning. Nueva York: Cambridge University Press.

Morales, J. (Coord.) (1994). Psicología social. Madrid: McGraw-Hill.

Morales, J. F., Maya, M., Gaviria, E., \& Cuadrado, G. (2007). Psicología social. Madrid: McGraw-Hill.

Myers, D. (2005). Psicología social. México: McGraw-Hill.

Oakes, J. (1985). The Distribution of Knowledege. Keeping Track: How Schools Structure Inequality. New Haven, CT: Yale University Press.

Pedulla, J., Airasian, P., \& Madaus, G. (1980). Do Teacher Ratings and Standardized Test Results of Students Yield the Same Information? American Educational Research Journal, 17, 303-307.
Ríos, C. (2006). Actitudes raciales en profesores de primaria y secundaria del distrito de Comas. Tesis presentada para optar por el título de licenciada en Psicología. Lima, Universidad Nacional Federico Villarreal.

Rosenthal, R. (1991). Meta-Analytic Procedures for Social Research (rev. ed.). Newbury Park, CA: Sage.

Rosenthal, R., \& Jacobson, L. (1968). Pygmalion in the Classroom: Teacher Expectation and Pupils' Intellectual Development. Nueva York: Holt, Rinehart and Winston.

Rosenthal, R. \& Jacobson, L. (1980). Pygmalion en la escuela. Madrid: Marova.

Rumelhart, D. E., \& Ortony, A. (1982). La representación del conocimiento en la memoria. Infancia y Aprendizaje, 19 (20), 115-118.

Woolfolk, A., Woolfolk Hoy, A., \& Margetts, K. (2010). Educational Psychology. Sidney: Pearson Education. 